\title{
Comparative analysis of simulation of urban radiation field
}

\author{
Dalong Liu ${ }^{1, *}$, Xiaowei Jia ${ }^{1}$, and Wenqin Wang ${ }^{2}$ \\ ${ }^{1}$ School of Architecture, Xi' an University of Architecture and Technology, Xi' an 710055, China \\ ${ }^{2}$ Library, Xi'an University of Architecture and Technology, Xi'an 710055, China
}

\begin{abstract}
In order to compare the differences between various outdoor environmental simulation software in the urban radiation field, ENVI-met, RayMan and SOLWEIG were selected as the research objects. The multiple types of urban radiation intensities that can be obtained by each software are compared, and the calculation method of types of these radiations was analyzed. Effects of underlying surface on the radiation field were simulated separately. The differences in radiation field performance simulations of three softwares were compared from several aspects such as short wave, direct radiation and reflected radiation. It is found that Rayman has the high sensitivity and fast simulation speed, but the output radiation parameters are less; ENVI-met can calculate the most radiation parameters and calculate the long-short wave radiation heat transfer process between building surfaces; the SOLWEIG can be used for simulation in large outdoor space, and has more calculated radiation parameters, but has large error. All three softwares can calculate the effect of space enclosure on direct and scattered radiation.
\end{abstract}

Key words.heat radiation; simulation of urban radiation field; performance comparison; underlying surface

\section{Introduction}

Solar radiation is a key factor affecting the urban thermal environment. Hardened underlying surfaces and high-density building groups cause not only direct radiation and short-wave scattered radiation, but also strong reflected radiation and long-wave radiation. The urban radiation field significantly affects the heat gain and loss of buildings. In addition, the urban radiation field has important effect on the thermal comfort of human body in outdoor environment. The complexity of urban radiation field in time and space inspired some scholars to investigate its trends by conducting tests. The tests include remote sensing observation [1], aerial observation [2], and ground observation [3]. These tests usually take long time and are affected by multiple aspects such as test field, time, and climate. Therefore, the reliability of measurement should be improved.

In recent years, simulation analysis has become the main approach in this field because of the advantages of consideration of many factors and high computation efficiency. By conducting a spot test, Song et al. [4] verified the accuracy of net radiation flux in an

* Corresponding author: coffeevc@xauat.edu.cn 
outdoor space and surface temperature simulated by ENVI-met. Matzarakis et al. [5] used the measured data to verify the simulation of short-wave and long-wave radiation flux in the 3D environment of RayMan; the total radiation and mean radiation temperature agreed well with the corresponding measured values. Dmitrieva et al. [6] simulated the mean radiation temperatures of a grand square and garth in Gothenburg, Sweden using SOLWEIG; the simulation results were consistent with the measured values.

the selection of a suitable calculation method and simulation software to achieve the expected simulation result has become an important issue, hindering the study of urban radiation field. In this study, widely used simulation softwares including ENVI-met, RayMan, and SOLWEIG were selected, and the performance of urban radiation field was compared by focusing on three respects: analog of radiation field and calculation model, simulation of effect of different underlying surfaces on radiation field, and effect of building envelopes on radiation field.

\section{Comparison of analog of radiation field and calculation models}

\subsection{Comparison in the analog of radiation field}

When performing the simulation calculation of a radiation field, the output analogs of radiation parameters of different software tools are different. The output analogs of radiation parameters of ENVI-met, RayMan, and SOLWEIG are shown in Table 1.

Table 1. Variation analysis in output radiation analog of each software

\begin{tabular}{|c|c|c|}
\hline ENVI-met & RayMan & SOLWEIG \\
\hline \multicolumn{3}{|c|}{ Short-wave radiation } \\
\hline Total radiation intensity & Total radiation intensity & Incident short-wave radiation intensity \\
\hline $\begin{array}{l}\text { Direct / Horizontal direct radiation } \\
\text { intensity }\end{array}$ & Direct radiation intensity & Direct radiation intensity \\
\hline Scattered radiation intensity & Scattered radiation intensity & Scattered radiation intensity \\
\hline \multirow{2}{*}{$\begin{array}{l}\text { Upper / lower hemisphere reflected } \\
\text { radiation intensity }\end{array}$} & & $\begin{array}{c}\text { Outward short-wave radiation } \\
\text { intensity }\end{array}$ \\
\hline & - & $\begin{array}{l}\text { Short-wave radiation intensity from } \\
\text { four different directions }\end{array}$ \\
\hline Surface long-wave radiation intensity & $\begin{array}{l}\text { Long-wave radiation } \\
\text { Atmosphere radiation intensity }\end{array}$ & 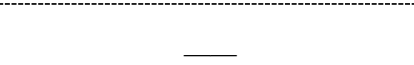 \\
\hline $\begin{array}{c}\text { Upper / lower hemisphere long-wave } \\
\text { radiation intensity } \\
\text { Long-wave radiation balance }\end{array}$ & (1) & $\begin{array}{l}\text { Incident / outward long-wave } \\
\text { radiation intensity }\end{array}$ \\
\hline- & - & $\begin{array}{l}\text { Long-wave radiation intensity from } \\
\text { four different directions }\end{array}$ \\
\hline Long-wave total flux & - & - \\
\hline
\end{tabular}

All three software tools can output the direct radiation quantity and scattered radiation quantity on a horizontal plane. ENVI-met and RayMan contain a radiation calculation model within themselves. By designating time, date, longitude, and latitude, specific position of the sun in the sky can be established, and the total solar radiation intensity, direct radiation intensity, and sky scattered radiation intensity of incidence to model boundary can be calculated. SOLWEIG has no built-in radiation calculation model. Therefore, the total radiation should be manually input in the calculation, but the total radiation can be divided into direct radiation and scattered radiation [7]. Incident shortwave radiation intensity in SOLWEIG can be used to consider the effects of buildings and plants on the total radiation intensity. In addition, SOLWEIG can calculate the short-wave 
radiation quantities for the vertical planes of four different directions: east, west, south, and north.

ENVI-met can output the reflected radiation intensity of a point in the space: In open space. SOLWEIG can also simulate reflection radiation intensity; outward short-wave radiation intensity considers the effects of buildings and plants on reflection radiation. In RayMan, the scattered radiation is a quantity that cannot be directly output, but the reflection radiation participates in the calculation of its radiation density [8]. ENVI-met can perform the output analog of underlying surface long-wave emission intensity, output of upper/lower hemisphere long-wave radiation flux. SOLWEIG can output the outward longwave radiation intensity; moreover, it can output long-wave radiation intensity from four different directions. RayMan can only output atmosphere long-wave radiation intensity.

Besides, the three software tools, ENVI-met, RayMan, and SOLWEIG, differ in the shielding treatment of radiation field. Shielding of radiation by an obstacle is represented by reduction factor in ENVI-met, and in the latest version, the IVS function considers the short- and long-wave radiation fluxes of multiple interactions between surfaces. In RayMan, the scattered radiation calculation is divided into two parts, namely, isotropic part and anisotropic part, conforming to the reality better than only considering the anisotropic part in ENVI-met. SOLWEIG uses the DEM of buildings and plants to establish a complex urban structure to calculate the radiation field. Among the three tools, ENVI-met can output the most radiation quantity, whereas RayMan can output the least radiation quantity.

\subsection{Comparison in radiation calculation model}

\subsubsection{ENVI-met}

ENVI-met is a simulation tool for urban microclimate, first developed by Michael Bruse in 1999. ENVI-met simulates the exchange of long-wave and short-wave radiations in urban. Multiple reduction factors between 0 and 1 are used in a radiation model to describe the shielding effects of buildings and plants on long-wave and short-wave radiations. The short-wave radiation flux of an arbitrary point in the region can be expressed as follows:

$$
Q_{s w}(z)=\sigma_{s w, d i r}(z) Q_{s w, d i r}^{0}+\sigma_{s w, d i f}(z) \sigma_{s v f}(z) Q_{s w, d i f}^{0}+\left(1-\sigma_{s v f}(z)\right) Q_{s w, d i r}^{0} \cdot \bar{a}
$$

where $\mathrm{Q}_{s w}(z)$ is the short-wave radiation flux of an arbitrary point in the region; $\sigma_{s w, d i r}(z)$ is the attenuation coefficient of obstacles to direct solar radiation; $\mathrm{Q}^{0}{ }_{s w, d i r}$ is the direct solar radiation intensity, $\mathrm{W} / \mathrm{m}^{2} ; \sigma_{s w, d i}(z)$ is the attenuation coefficient of obstacles to diffuse solar radiation; $\mathrm{Q}_{s w, d i f}^{0}$ is the diffuse radiation intensity, $\mathrm{W} / \mathrm{m}^{2} ; \sigma_{s v}(z)$ is the sky angular coefficient; ,$a$ is the mean reflectivity of all the walls in the model region.

\subsubsection{RayMan}

RayMan (Radiation on the human body) is a tool for evaluating the thermal radiation of human body, developed by Prof. Matzarakis from University of Freiburg, Germany, in 2007. RayMan can be used to calculate the effects of elements such as terrain, buildings, and plants on the radiation flux in a complex environment. The sensitivity of RayMan is extraordinarily high, and even when meteorological file is not available, the results can be quickly obtained using simple parameters; therefore, RayMan can be applied in urban design [9].

When calculating direct radiation, the radiation intensity in RayMan can be calculated as follows: 


$$
I=I_{0} \cdot \cos \zeta \cdot \exp \left(-T_{L} \cdot m_{r 0} \cdot \frac{\rho}{\rho_{0}}\right) \cdot\left(1-\frac{N}{8}\right)
$$

where $I$ is the direct radiation intensity, $\mathrm{W} / \mathrm{m}^{2} ; I_{0}$ is the solar radiation intensity vertical to the plane of incidence direction, $\mathrm{W} / \mathrm{m}^{2} ; \zeta$ is the zenith angle, deg; $T_{\mathrm{L}}$ is the Linke turbidity factor; $m_{r 0}$ is the vertical optical depth of standard Rayleigh atmosphere, $\mathrm{m} ; \rho$ is the local atmospheric pressure,hPa; $\rho_{0}$ is the standard atmospheric pressure, $\rho_{0}=1013 \mathrm{hPa} ; N$ is the turbid degree.

\subsubsection{SOLWEIG}

SOLWEIG (Solar and Longwave Environmental Irradiance Geometry) is a tool for estimating the spatial variation of $3 \mathrm{D}$ radiation flux and mean radiation temperature in urban, developed by the urban climate team of University of Gothenburg in Sweden in 2008. This tool constructs complex urban structures through the digital elevation model (DEM) of buildings, plants, etc.; therefore, the simulation has a relatively high accuracy [10].

The solution of direct radiation in SOLWEIG is expressed as follows:

$$
I=\frac{G-D}{\sin \eta}
$$

where $I$ is the direct solar radiation intensity, $\mathrm{W} / \mathrm{m}^{2} ; G$ is the total radiation intensity, $\mathrm{W} / \mathrm{m}^{2} ; D$ is the scattered radiation intensity, $\mathrm{W} / \mathrm{m}^{2} ; \eta$ is the solar elevation angle, deg.

\section{Test verification of simulation results}

\subsection{Conditions of test and simulation}

To verify the accuracy of different tools simulating an urban radiation field, the radiation data of above different underlying surfaces measured in Xi'an of China on 11th, March 2018. The field test period was 8:30-17:30. The total horizontal radiation intensity, horizontal direct radiation intensity, horizontal scattered radiation intensity, and reflection radiation intensity of the location $1.1 \mathrm{~m}$ above three different ground surfaces were measured once every $30 \mathrm{~min}$.

The versions of simulation tools were ENVI-met4.31, RayMan1.2, and SOLWEIG 2015a. The geographic locations of tools were unified as Xi'an. The solar radiations of ENVI-met and RayMan were generated using a built-in radiation loading model, whereas SOLWEIG used the total radiation data simulated by RayMan. When simulating the radiation field, the spatial point $1.1 \mathrm{~m}$ above different underlying surfaces was selected for the comparison of radiation intensity.

\subsection{Comparison in solar direct, scattered, and reflection radiation intensities}

Fig. 1 shows the variation curves of direct radiation intensity of horizontal plane for a spatial point $\mathrm{M}$ above different underlying surfaces in a day simulated by three tools. Because of no shielding of obstacles such as buildings and plants above different underlying surfaces, the direct radiation intensities of point $\mathrm{M}$ above the three underlying surfaces had no change. The direct radiation intensities simulated by the three tools and that measured reached their strongest radiation intensities all at noon; they are relatively weak in 
the morning and at dusk in a day. Regarding scattered radiation, the scattered radiation conditions of three underlying surfaces in a day are shown in Fig. 2. No variation in scattered radiation occurred across different underlying surfaces because the gain of scattered radiation produced by surface reflection radiation was not considered by any of the three tools in scattered radiation calculation. Among these tools, the value calculated using ENVI-met is the closest to the measured value, followed by RayMan. There is a large difference between the SOLWEIG and measured values.

The reflection radiation intensities of point $M$ above concrete, brick pavement, and asphalt surfaces are shown in Figs. 3-4 below. Because RayMan cannot directly output reflection radiation intensity, it is not included in the comparisons. conforming to the measured pattern, and ENVI-met showed results closer to the measurement.

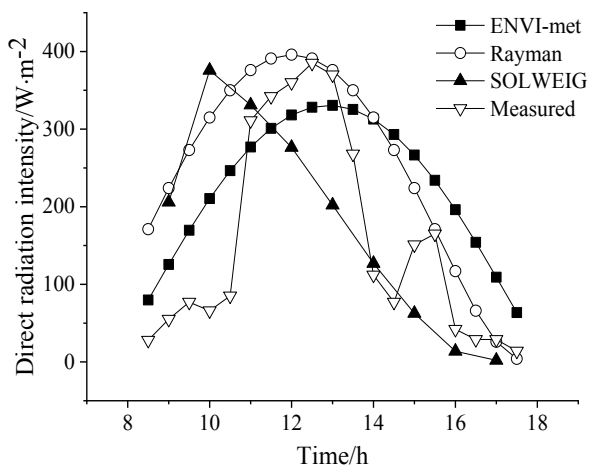

Fig. 1. Direct radiation intensity

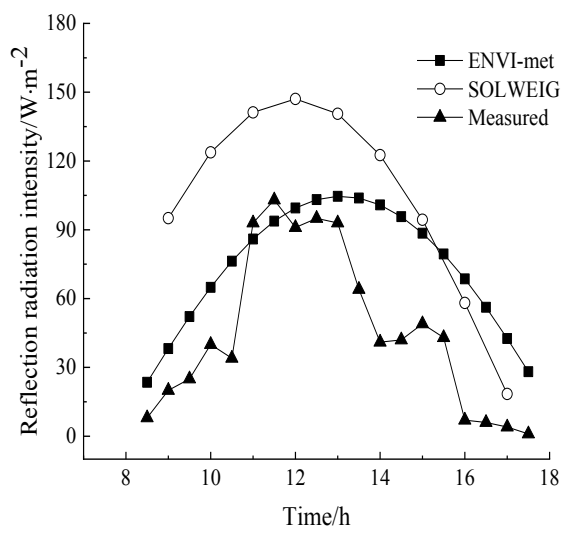

Fig. 3. Reflection radiation of concrete surface

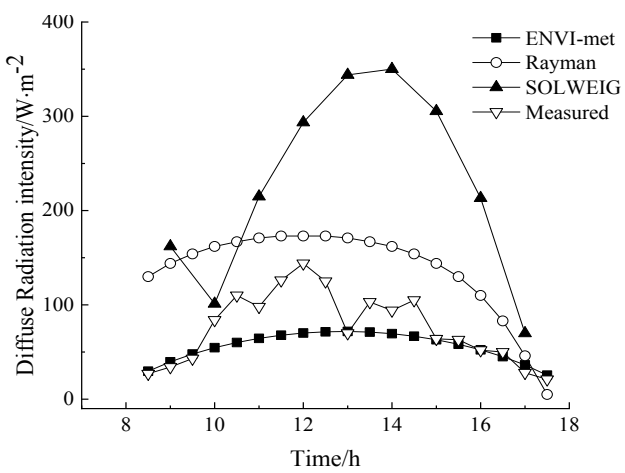

Fig. 2. Diffuse radiation intensity

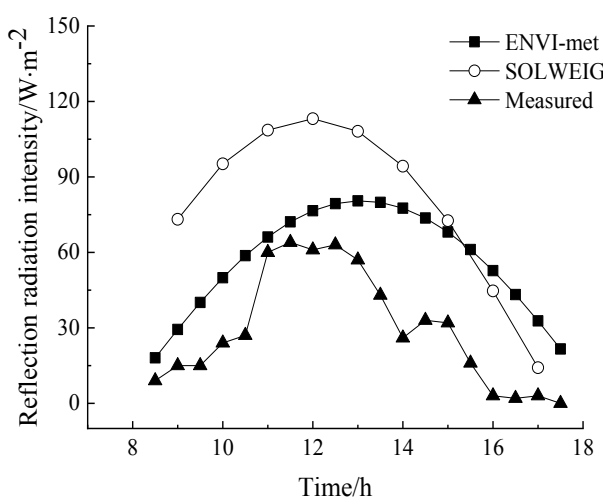

Fig. 4. Reflection radiation of pavement brick

According to the comparison of radiation simulation for different underlying surfaces, a good consistency was observed among the values simulated by three tools and the corresponding measured values regarding direct, scattered, and reflection radiation intensities of different underlying surfaces. However, certain differences exist when comparing the strengths of radiation intensities among the simulations of three tools and measurement; this may be caused by the complexity of test environment and insufficiency of consideration of urban radiation within the models. 


\section{Conclusions}

The simulation of urban microclimate has become increasingly important to not only building energy conservation, but also landscape design and even to urban planning. To distinguish the performance difference among ENVI-met, RayMan, and SOLWEIG on simulating urban radiation field, first the corresponding main radiation calculation models were compared. Then, by focusing on the effects of different underlying surfaces on a radiation field in an open space, based on direct radiation, scattered radiation, reflection radiation, etc., combined with the measured radiation data, the three tools were compared and analyzed. that the following observations were made: RayMan has a high calculation sensitivity and fast simulation speed, but the output radiation parameters are relatively few. ENVI-met can calculate the most number of radiation parameters, and it considers the radiation heat transfer of short waves between building surfaces. SOLWEIG can be applied for outdoor large-space simulation, and many calculated radiation parameters are present, but the error is relatively large. All the three software tools consider the effects of space enclosure on direct radiation and scattered radiation.

This work was supported by the National Natural Science Foundation of China (Grant No. 51578439) and the Major Program of the National Natural Science Foundation of China (Grant No. 51590913) and the State Key Laboratory of Subtropical Building Science (South China University of Technology, Grant No.2018ZB16)

\section{References}

[1] C. M. Frey, G. Rigo, E.Parlow. INT.J.REMOTE.SENS 28(12): 2695-2712(2007)

[2] H. Sugawara, T.Takamura. BOUND-LAY.METEOROL 153 (3): 539-553(2014).

[3] PENG J L, WU X, LIU H N. S\&TR 26(3):34-40(2008).

[4] B.G.Song, K.H.Park, S. G. Jung. J.Korean.Assoc.Geogr.Inf.Stud 17(2): 156-172(2014).

[5] A.Matzarakis, F. Rutz, H.Mayer. INT.J.BIOMETEOROL 51(4): 323-334(2007).

[6] T. B. Dmitrieva, A.V.Kirenskaya,V. Y.Novototsky-Vlasov.et al. INT.J.PSYCHOPHYSIOL 69(3): 200(2008).

[7] D. T.Reindl, W.A. Beckman, J.A.Duffie. SOL.ENERGY 45(1): 1-7(1990).

[8] A.Matzarakis,F. Rutz, H. Mayer. INT.J.BIOMETEOROL 54(2): 131-139(2010).

[9] A. Matzarakis,F.Rutz. 13.2 9UES,(2010).

[10] K.Hammerberg,A.Mahdavi. ECPPM 2014 243(2014). 